\title{
Vogt-koyanagi-harada syndrome in a young patient
}

\begin{abstract}
Vogt-Koyanagi-Harada syndrome is a chronic bilateral autoimmune intraocular inflammation in which antibodies are produced against melanocyte tissues associated with neurological and skin changes, including headache, tinnitus, vitiligo, polyiosis, and alopecia. The disease occurs in distinct phases: the prodromal phase, the acute uveitic phase, the chronic or convalescent phase, and the chronic recurrent phase. The treatment should be early and is made using corticosteroids and immunomodulatory drugs. We report a case of a young patient with the syndrome who developed recurrence of the disease during oral corticosteroid weaning, and was treated with adalimumab.
\end{abstract}

Keywords: vogt-koyanagi-harada syndrome, panuveitis, exudative retinal detachment

\author{
Volume 4 Issue 4 - 2020
}

\author{
Lorena Ribeiro Ciarlini,Aline Barbosa \\ Pinheiro Bastos, Alana Andrade Neiva Santos, \\ Ingrid Cavalcante Sarquis, Raissa Oliveira \\ Campos, Fernanda Araújo Souza,Vitor Cruz \\ Furtado,Alexis Galeno Matos \\ Leiria de Andrade Foundation Fortaleza/Ceara, Ribeirão Preto \\ Medical School, University of São Paulo, Brazil
}

Correspondence: Alexis Galeno Matos, Leiria de Andrade Foundation - Fortaleza/Ceara, Ribeirão Preto Medical School, University of São Paulo, Brazil, Tel 5585996852005.

Email alexisgleno@gmail.com

Received: July 03, 2020 | Published: July 17, 2020

\section{Introduction}

Vogt-Koyanagi-Harada syndrome (VKH), initially described as uveomeningoencephalitic syndrome, is a set of signs and symptoms that involve several organs. ${ }^{1}$ It was Vogt in 1906 who reported a case of previous uveitis associated with poliosis and dysacusis and in 1926 Harada described the presence of posterior uveitis with exudative retinal detachment, associated with meningeal signs and cerebrospinal fluid pleocytosis. ${ }^{2}$ In 1929, Koyanagi described patients with bilateral iridocyclitis associated with alopecia and vitiligo, deafness and tinnitus when in the 1940s, Babel recognized VKH syndrome or uveomeningeal syndrome as a single entity. ${ }^{2,3}$ The syndrome is a bilateral chronic intraocular inflammation associated with neurological and skin changes, including headache, tinnitus, vitiligo, poliosis and alopecia. Visual impairment with headache is the most prevalent complaint and vitreous and anterior chamber cellularity are the most common signs of acute VKH. This condition represents about $2 \%$ of the cases referred to the uveitis department. ${ }^{4}$ It affects adults of both sexes, with a predilection for the female sex, between 20 and 50 years old, and for the black race, oriental and Hispanic. ${ }^{3}$ It is considered a systemic autoimmune disease characterized mainly by bilateral granulomatous panuveitis, in which antibodies are produced against tissues rich in melanocytes. ${ }^{5}$

The response would be mediated by $\mathrm{T}$ lymphocytes and directed against melanocytic antigens in the eye (specifically the retina and the iris), skin, nervous system and inner ear. ${ }^{2}$ Possible targets include antigenic tyrosinase and protein S-1001. In electron microscopy studies, they observed intimate contact between melanocytes and lymphocytes of the choroidal basement membrane. ${ }^{6}$ In the acute phase, a preponderance of CD4 + lymphocytes was observed, while in the convalescence phase it was of CD $8+.^{7}$ There is an association with different HLA haplotypes, and the most frequent is HLA-DR4. Recently, a new autoantigen of the syndrome, KU-MEL-1, forming part of HLADRB1, which could be used for the diagnosis of disease. ${ }^{8}$ The disease occurs in different phases: the prodromal phase, the acute uveitic phase, the chronic or convalescent phase, and the chronic recurrent phase. In the prodromal phase, the symptoms are nonspecific, including fever, headache, nausea, meningism, vertigo and dysacusis.
They rarely include ataxia, confusion and focal neurological disorder. It usually lasts 3 to 5 days. ${ }^{5}$ The acute uveitic phase begins a few days after the prodromal phase and can last between 2 and 3 months. It is characterized by bilateral uveitis, although in some cases, one eye may be affected first and subsequently the other, with the sudden appearance of blurred vision, photophobia and eye pain. It may be accompanied by dysacusis and meningism. ${ }^{3}$ Choroiditis also occurs, which results in rupture of the external hematoretinal barrier, causing exudative retinal detachment. In addition, it can cause acute glaucoma. ${ }^{5}$ At this stage, some endocrinological disorders are found, such as, amenorrhea, pituitary dysfunction, diabetes insipidus, hyperglycemia and hypocholesterolemia. ${ }^{3}$

The next phase is the chronic phase or convalescence, where the retinal detachment gradually regresses and uveitis begins to decrease. There is a pigmentary redistribution that gives rise to an ocular fund at "sunset". This phase can take months. ${ }^{5}$ There are also changes described in the retinal pigment epithelium (pigment redistribution that forms images similar to the nevus) and atrophic areas. In the sclerocorneal limbus, you can find a depigmentation known as the "Sugiura sign". At this stage, skin symptoms, poliosis, alopecia and vitiligo are frequent, in addition to paresthesias and dysesthesias. It frequently affects the face, neck, trunk and eyelids. ${ }^{3}$ The last phase is the chronic recurrent that occurs months to years after the acute phase, interrupting the convalescence phase. This phase is not present in all patients. It is characterized by episodes of granulomatous anterior uveitis. It is the stage of chronic complications, such as cataracts, glaucoma, macular edema, choroidal and papillary neovascularization and neovascular subretinal membrane. ${ }^{3}$

The diagnosis of VKH syndrome is established by clinical criteria of the American Uveitis Society (Table 1). It is defined as complete syndrome when it meets criteria 1 to 5 , incomplete when it meets criteria 1 to 3 , plus the fourth or fifth criterion, and probable when it presents only ocular criteria from 1 to 3 . Additional exams are not necessary, but provide greater diagnostic security. Ocular ultrasound helps when opaque media are present, showing posterior choroidal thickening and serous detachment of the retina. The initial phase of fluorescein angiography has several hyperfluorescent spots in the 
pigment epithelium. The lumbar puncture shows cerebrospinal fluid pleocytosis with a predominance of lymphocytes. Magnetic resonance imaging allows to differentiate this disease from posterior scleritis.
Optical coherence tomography is useful to quantify the evolution of serous detachment, for diagnosis and monitoring of complications such as macular edema and neovascular membranes.

Table I American Uveitis Society clinical criteria for diagnosingVKH syndrome

I.Absence of penetrating eye trauma or surgery preceding the onset of uveitis

2. Absence of clinical or laboratory evidence of another eye disease

3. Bilateral eye involvement (criteria A or B must be met according to the stage at which the patient is examined)

A . Early manifestations of the disease:

Evidence of diffuse choroiditis (focal areas of subretinal fluid or bullous / serous retinal detachments);

If fundscopic findings are doubtful, both of the following must be present: focal areas of choroidal perfusion delay, focal areas of punctiform overflow, placoid areas of hyperfluorescence, damming of subretinal fluid and enhancement of the optic nerve (listed following appearance) fluorescein angiography; and

Diffuse choroidal thickening without evidence of posterior scleritis by ultrasound.

B. Late manifestations of the disease:

Previous history of the presence of $3 \mathrm{~A}$ findings;

Eye depigmentation: sign of sigilitude (perilimbic vitiligo) or background in sunset glow (sunset glow);

Other ocular signs, such as: depigmented chorio-retinal numeral scars; or migration or clusters of the retinal pigmented epithelium; or chronic or recurrent anterior uveitis.

Neurological or auditory findings (which may have resolved at the time of the exam)

Meningism (asthenia, fever, headache, nausea and stiff neck or a combination of these factors; headache alone is not sufficient for its definition); or Tinnitus (tinnitus), or cerebrospinal fluid pleocytosis

Dermatological findings (should not precede the onset of neurological or ophthalmic symptoms)

\begin{tabular}{ll} 
- & Alopecia; or \\
- & Poliosis; or \\
\hline
\end{tabular}

Source: Frutos et al (2012)

Approximately $50 \%$ of patients have complications such as cataracts; glaucoma, either from a closed angle, due to anterior displacement of the iris-crystalline complex or by the formation of peripheral anterior synechiae, or from an open angle, due to the prolonged use of glucocorticoids. Choroidal neovascular membrane, subretinal fibrosis and extensive retinochoroidal atrophy can also be found. ${ }^{3}$ Different drugs are used in the treatment of recurrent VKH, with varying efficiency. Systemic glucocorticoids are the drug of choice in acute outbreaks. The initial dose, duration of treatment and the descending pattern must be individualized in each patient. Various medications, such as immunosuppressants, are also used. Biological drugs and immunoglobulins. Glucocorticoids are used mainly in the acute phase to prevent decreased visual acuity. Early administration can prevent disease progression, shorten its duration and decrease systemic involvement. ${ }^{3}$ Among immunosuppressants, different drugs have been used with varying efficacy, including cyclosporine A, chlorambucil, cyclophosphamide, tacrolimus, mycophenolate, azathioprine, methotrexate and even intravenous immunoglobulins. ${ }^{3}$ Several studies have considered the efficacy of adalimumab in treating patients with chronic uveitis refractory, in addition to allowing a reduction in the use of glucocorticoids. ${ }^{9} 10$ Jaffe et al demonstrated that treatment with adalimumab effectively achieves early and sustained control of the disease after discontinuation of the glucocorticoid by reducing inflammation and reducing visual impairment in patients with intermediate uveitis, posterior uveitis, or panuveitis. ${ }^{11}$

\section{Case report}

A.E.C.F., 29 years old, male, was seen complaining of low visual acuity in his left eye for 3 days. Patient without comorbidities, did not use routine medication and had no family history of ophthalmic diseases and trauma. In the first evaluation, she presented: visual acuity (without correction) of the right eye (RIGHT EYE): 20/25 and left eye (LEFT EYE): 20/70. In the biomicroscopy of the anterior segment, the right eye was unchanged; left eye with hyperemia of conjunctiva $2+/ 4+$, transparent cornea, anterior chamber formed, reaction in anterior chamber $2+/ 4+$, flare $1+/ 4+$ and posterior synechiae. Intraocular pressure by Goldmann applanation tonometer, RIGHT EYE: $12 \mathrm{mmHg}$, LEFT EYE: $22 \mathrm{mmHg}$. Retinography of the right eye showed a stained optic nerve with physiological excavation, a macula without changes, an applied retina and a healed toxoplasmosis plaque in the temporal retinal region (Figure 1). Laboratory tests were requested, including serology for infectious diseases, and eye drops of prednisolone $1 \%$ at $6 / 6 \mathrm{~h}$ with weekly weaning and tropicamide $1 \%$ at $8 / 8 \mathrm{~h}$ were started in the left eye. The patient returned after 15 days reporting improvement in hyperemia and pain in the left eye, but with worsening visual acuity in both eyes: 20/100 in RE and figures in LE.

Laboratory tests showed the following results: complete blood count without changes, serology for non-reactive HIV, VDRL $1 / 128$, toxoplasmosis IgG positive (185.0) and IgM negative (0.43), serologies for hepatitis $\mathrm{B}$ and $\mathrm{C}$ negative, FAN negative and negative 
rheumatoid factor. Ultrasonography was then performed in both eyes, which showed the presence of multiple pockets of serous retinal detachment with a macula glued to the right eye; and multiple pockets of serous retinal detachment, with macular edema in the left eye. With the diagnosis of Vogt-Koyanagi-Harada syndrome closed, treatment with oral prednisone $1 \mathrm{mg} / \mathrm{kg}$ was initiated, in addition to maintaining $1 \%$ prednisolone eye drops at weekly weaning. Two weeks after the beginning of oral immunosuppressive therapy, there was an improvement in visual acuity, which presented, with correction, in DO, 20/30 and in LEFT EYE, 20/40; in addition to reduction of retinal edema. Intraocular pressure using Goldmann's tonometry, OD $24 \mathrm{mmHg}$ and LEFT EYE $26 \mathrm{mmHg}$, and associated brimonidine eye drops with timolol of $12 / 12 \mathrm{~h}$ in both eyes (BE). In subsequent consultations, the patient presented negative VDRL after treatment for syphilis and control of intraocular pressure using hypotensive eye drops. Slow weaning from oral prednisone was carried out over the course of a year and maintained at $20 \mathrm{mg} /$ day with stabilization of visual acuity at 20/20, with correction, in both eyes. One year after the onset of the condition, the patient presented to the retinography the presence of a "sunset" image in the right eye (Figure 3a) and in the left eye (Figure 3b) Angiography showed the presence of hyperfluorescent lesions due to a window defect, due to the mobilization area and atrophy of the retinal pigment epithelium, seen during the examination, both in the left eye (Figure 5a) and in the right eye (Figure $5 b$ ). In an attempt to completely withdraw oral medication with slow weaning, visual loss of three lines was observed in the Snellen table in RIGHT EYE (20/40) and LEFT EYE remained stable at $20 / 20$. Thus, a dose of $20 \mathrm{mg}$ / day of oral prednisone was maintained for two months, with slow weaning to $5 \mathrm{mg} /$ day, but again there was a worsening of VA (RIGHT EYE: 20/40 and LEFT EYE: 20/30). Thus, it was decided to suspend oral prednisone at $20 \mathrm{mg} /$ day and start injectable treatment with adalimumab. The patient is waiting for the medication to be released by the Unified Health System.
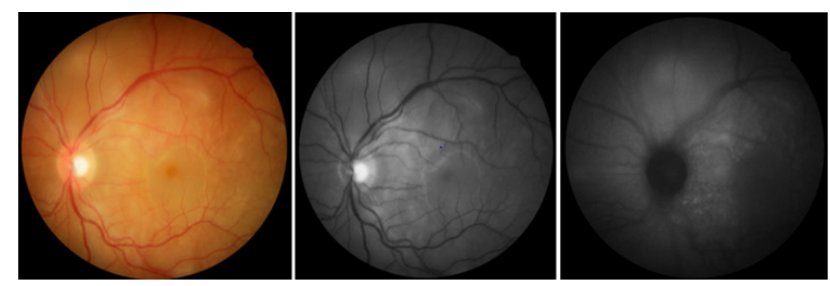

Figure I Retinography of the right eye in 2018.

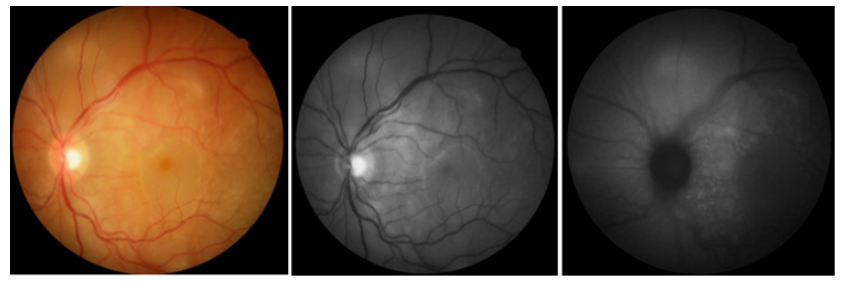

Figure 2 Retinography of the left eye in 2018.
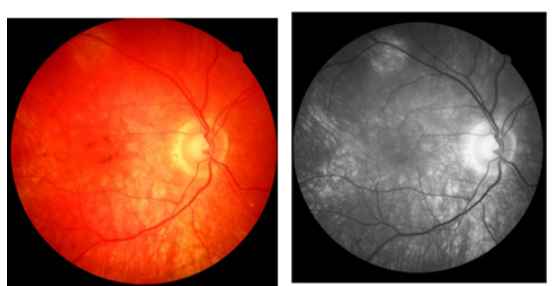

Figure 3a Retinography of the right eye.

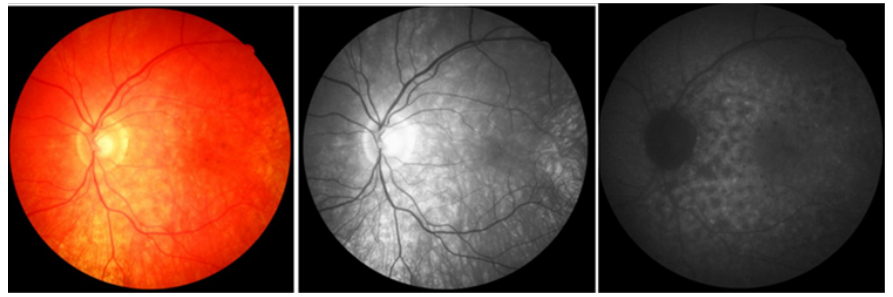

Figure 3b Retinography of the left eye.

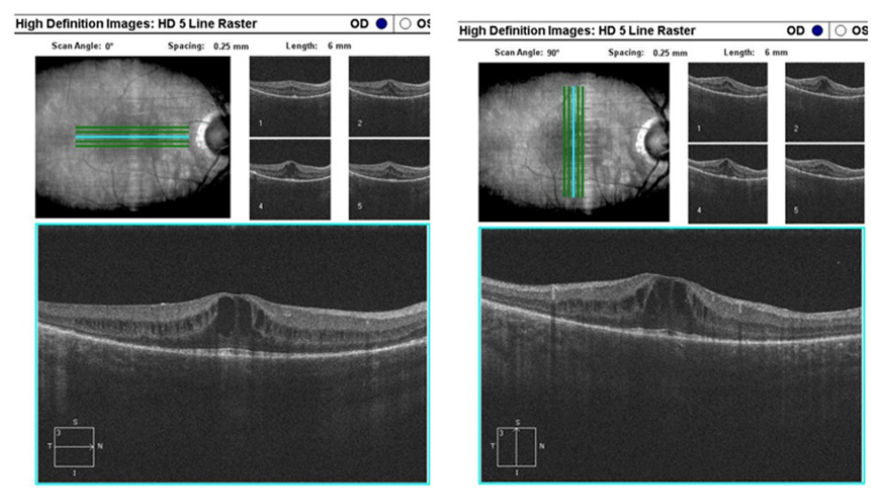

(a) - right eye

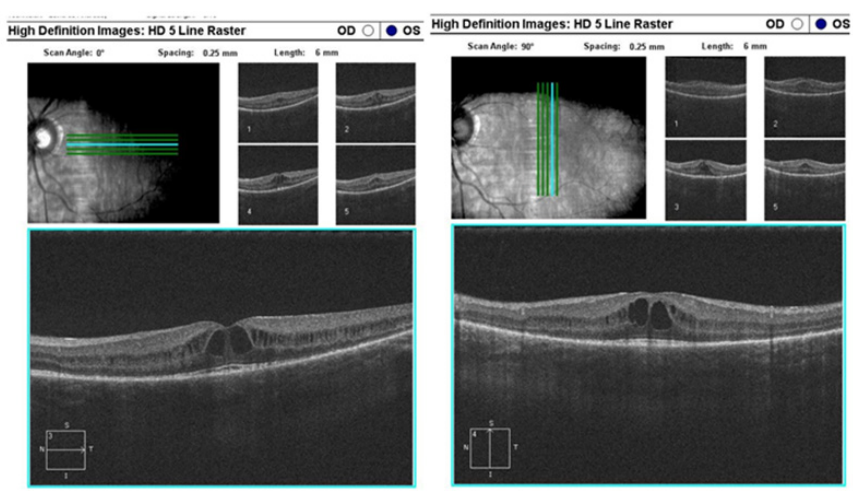

(b) - left eye

Figure 4 Optical coherence tomography.
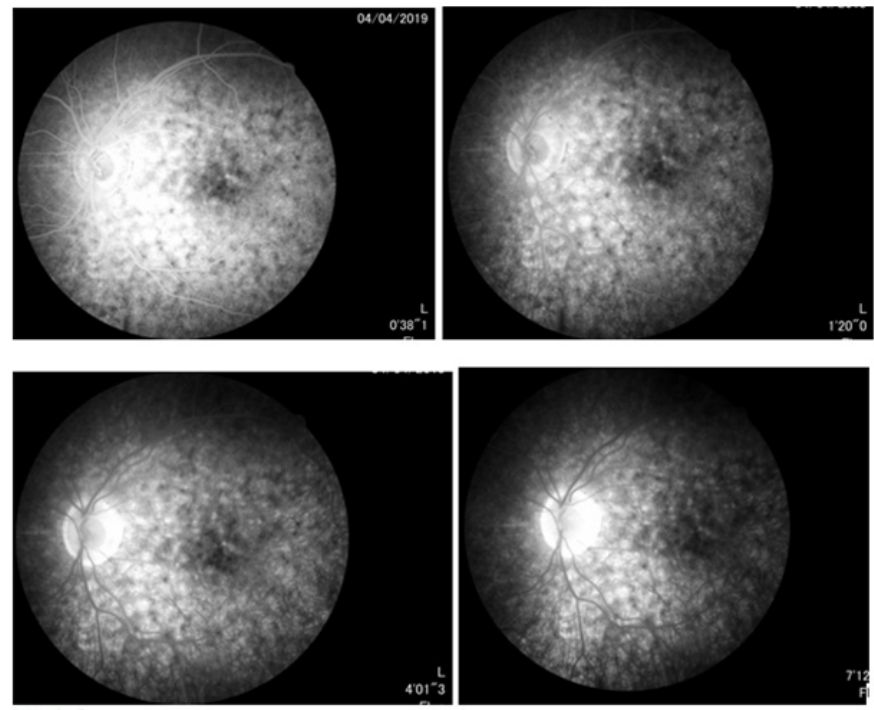

a)-left eye 

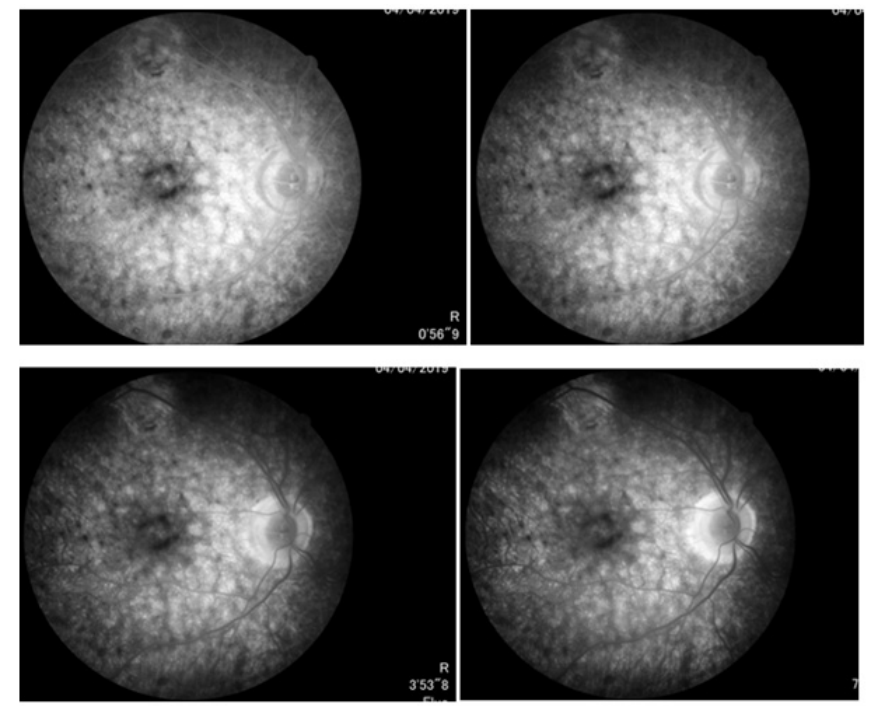

b) - right eye

Figure 5 Fluorescein angiography.

\section{Discussion}

VKH syndrome is a severe bilateral granulomatous panuveitis that threatens eye health. It is believed to be an autoimmune disorder. ${ }^{12}$ The disease presents a variable ocular and systemic clinical picture, and the clinical diagnosis is challenging, since it involves a multidisciplinary medical team. ${ }^{1}$ The differential diagnosis is made with infectious diseases of neurological, ophthalmological and cutaneous involvement, such as syphilis, toxoplasmosis and AIDS, ${ }^{13}$ as well as non-infectious pathologies such as posterior scleritis and sympathetic ophthalmia that is related to eye trauma. ${ }^{5}$ Panuveitis is the most common sign found and the key to the diagnosis of this condition. The most common ocular signs of VKH syndrome are iridocyclitis, vitreitis, edema or hyperemia of the optic disc, choroidal thickening and neuro-sensory retinal detachment. ${ }^{14}$ The described report shows the case of a patient who started the condition with unilateral symptoms in addition to macular edema in one eye, evolving with serous detachment and low bilateral visual acuity. Four clinical stages have been described for this condition. In the prodromal phase, meningoencephalic involvement occurs, mimicking a viral infection that lasts from days to weeks. This phase occurs in about $50 \%$ of cases. ${ }^{14,15}$ The patient described in the case reported having headache, nausea and vertigo days before the ocular condition, but that she thought she was part of a migraine attack. The acute uveitic phase begins about three to five days after the prodromal phase and lasts a few weeks. The most common symptoms are: visual turbidity, eye pain and low visual acuity secondary to posterior uveitis with retinal edema, optic disc edema, retinal serous detachments, iridocyclitis and increased intraocular pressure. In this phase, auditory alterations also occur. ${ }^{15}$ In the reported patient, all of these most common eye symptoms have been described.

Two months after the onset of the condition, dermatological changes may also appear, constituting the convalescent. They are poliosis, vitiligo and alopecia. However, the study patient did not present any evident dermatological symptoms. The characteristic " sunset " eye fundus was described in the assessment made one year after the onset of the condition. ${ }^{15}$ The recurrent chronic phase may or may not occur. When present, recurrent retinal detachments, formation of retinal neovascular membranes, glaucoma and cataracts occur. These complications occur in about $1 / 3$ of the patients. ${ }^{14,15}$ Our patient progressed well with treatment and did not show recurrence symptoms, despite the difficulty in weaning from therapy and staying on medication for more than a year. Regarding the most used complementary exams, in the OCT the most common findings are folds and serous detachment of the retinal pigment epithelium (RPE), presence of septa and areas of subretinal fluids, with macular area thickness varying between 700-900 microns ( 12.16). Ocular ultrasound can show choroidal thickening, papilla edema and areas of serous retinal detachment. Regarding fluorescein angiography, fluorescein pooling can be seen in areas of serous detachment and leakage of the optical disc. ${ }^{12}$ In the patient, the OCT (one year after the onset of the condition) showed an increase in foveal thickness and cysts in the internal retinal layers; on ultrasound, we see the presence of multiple pockets of serous retinal detachment; and in fluorescein angiography, we observed hyperfluorescent areas by window and pooling defect. Such findings corroborate the evidence in the literature cited above. Treatment must be early and it is done with the use of corticosteroids and immunomodulatory drugs to control the effects produced by autoimmunity and reduce damage. ${ }^{1}$ After the symptoms improve, the treatment should be discontinued slowly, from 3 to 6 months, and should be maintained for about a year in case of recurrences. In severe forms of the disease, there is benefit in the triple use of immunosuppressive therapy, which consists of prednisolone, azathioprine and cyclosporine, simultaneously. ${ }^{1}$ The inflammatory and exudative retinal process can improve with the application of intravitreal triamcinolone injection. ${ }^{17}$ Some studies suggest that sub-tenonian injection of triamcinolone should be an initial treatment for relapse of VKH in pregnant women. ${ }^{18}$ The study patient improved only with prolonged use of oral and topical corticosteroids, not requiring immunomodulatory therapy. Poor access to immunomodulatory medications through the Unified Health System also restricts treatment to corticosteroids. The main complications of VKH syndrome are secondary glaucoma, cataracts, choroidal neovascularization and subretinal neovascular membrane in the recurrent chronic phase. ${ }^{14,19}$ The study patient did not have any of these complications. Chronicity of the condition usually occurs when there is a delay in diagnosis, often due to ignorance of the initial clinical manifestations of the disease. The time between the onset of symptoms and the institution of immunosuppressive therapy is essential for final visual acuity, influencing the prognosis of the disease.

\section{Conclusion}

Although rare, VKH syndrome requires accurate and early diagnosis to avoid complications and major visual loss. As it is a pathology with bilateral involvement in most cases, it is responsible for causing visual impairment in many underdiagnosed or poorly conducted cases. It is also essential to differentiate from other pathologies that occur with serous detachments of the retina, such as sympathetic ophthalmia, posterior scleritis and central serous chorioretinopathy, considering that they present different pathophysiology and differentiated treatments. The multisystemic involvement of the disease, including dermatological, neurological and ophthalmological changes, requires a trained multidisciplinary team to properly manage the therapy and improve the visual prognosis. Because the treatment involves immunosuppressive medications for long periods, the patient must be closely monitored for possible adverse effects, as well as periodic examinations in order to avoid aggravations due to the use of immunosuppressants. 


\section{Acknowledgments}

None.

\section{Conflicts of interest}

The author declares there is no conflcit of interest.

\section{References}

1. Marcelo TT, Iguban EB. Case Report A Case Report on Vogt-KoyanagiHarada Disease Seen at a Tertiary Hospital in the Philippines. The ophthalmology open journal. 2018;3(1):1-4.

2. M GILH, P AR, A HB, et al. Evolución severa en Enfermedad de VogtKoyanagi-Harada Severe evolution of Vogt-Koyanagi-Harada disease. ARCH SOC CANAR OFTAL. 1998;51-54.

3. Riveros A, Romera P, Holgado S, et al. Seminarios de la Fundación Española de Reumatología. Enfermedad de Vogt-Koyanagi-Harada. 2012;13(4):142-146.

4. Singh S, Kaushik S, Kaur S, et al. Vogt-Koyanagi-Harada syndrome presenting as bilateral simultaneous acute angle closure. Clinical and Experimental Vision and Eye Research. 2019;2:49-51.

5. Lavezzo MM, Sakata VM, Morita C, et al. Vogt-Koyanagi-Harada disease : review of a rare autoimmune disease targeting antigens of melanocytes. Orphanet J Rare Dis. 2016;1-21.

6. Hayakawa K, Ishikawa M, Yamaki K. Ultrastructural Changes in Rat Eyes with Experimental Vogt-Koyanagi-Harada Disease. Laboratory Investigation. 2004;222-227.

7. Umihira J, Seki A, Nohara M, et al. Dominance of Activated T Cells and Interleukin-6 in Aqueous Humor in Vogt-Koyanagi-Harada Disease. IOVS. 1994;35(1):33-39.

8. Takeuchi M, Kawakami Y. Frequent immune response to a melanocyte specific protein KU-MEL-1 in patients with Vogt-Koyanagi-Harada disease. Br J Ophthalmol. 2006;90(6):773-777.
9. Díaz-llopis M, Salom D, Garcia-de-vicuña C, et al. Treatment of Refractory Uveitis with Adalimumab : A Prospective Multicenter Study of 131 Patients. OPHTHA. 2012;119(8):1575-1581.

10. Dobner BC, Max R, Becker MD, et al. A three-centre experience with adalimumab for the treatment of non-infectious uveitis. The British Journal of Ophthalmology. 2013;90(2):134-138.

11. Adalimumab EB, Uveitis N, England N, et al. Adalimumab in Patients with Active Noninfectious Uveitis. The new England Journal of Medicine. 2016;375:932-943.

12. Pellegrini F, Interlandi E, Prosdocimo G. Vogt-Koyanagi-Harada Disease Presenting as Unilateral Neuroretinits. Neuro-Ophthalmology. 2017;42(1):1-6.

13. Rao NA, Sukavatcharin S, Tsai JH. Vogt-Koyanagi-Harada disease diagnostic criteria. Int Ophthalmol. 2007;27(2-3):195-199.

14. Almeida M, Baptista N, Veríssimo J, et al. Doença de Vogt-KoyanagiHarada : A propósito de 30 Casos Clínicos. Rev SPO. 2011;35:33-41.

15. Read RW, Holland GN, Rao NA, et al. Revised diagnostic criteria for Vogt-Koyanagi-Harada disease: Report of an international committee on nomenclature. Am J Ophthalmol. 2001;131(5):647-652.

16. Tufaili HA. A Misleading Presentation of Vogt - koyanaji - Harada Disease. Karbala J Med. 2018;11(2):4059-4063.

17. Andrade REA, Muccioli C, Farah ME. Injeção intravítrea de acetato de triancinolona no tratamento da síndrome de Vogt-Koyanagi-Harada. Arq Bras Oftalmol. 2004;67(3).

18. Nakamura T, Keino H, Okada AA. Sub-Tenon triamcinolone acetonide injection in a pregnant patient with Vogt-Koyanagi-Harada disease. Retin Cases Br Reports. 2018;12(4):375-378.

19. Mota LAA, dos Santos AB. Síndrome de vogt-koyanagi-harada e o seu acometimento multissistêmico. Rev Assoc Med Bras. 2010;56(5). 